\title{
Development and Practice of Integrated Nursing Model NNN Link for Breastfeeding Clinical Nursing Model as a Whole
}

\author{
Xiaoqin Guo \\ Lecturer of the Graduate School, Shanxi Medical University, Taiyuan, China \\ Email: gxq1970@126.com \\ Received 18 December 2015; accepted 15 February 2016; published 18 February 2016 \\ Copyright (C) 2016 by author and Scientific Research Publishing Inc. \\ This work is licensed under the Creative Commons Attribution International License (CC BY). \\ http://creativecommons.org/licenses/by/4.0/

\section{Abstract}

Objective: To establish breastfeeding clinical holistic nursing model used in clinical nursing and teaching, it is possible to improve nurses and nursing students to master knowledge of breastfeeding, analysis, judgment, decision-making and the ability to care-related issues, promoting pure improve breastfeeding rates. Method: 1) The North American Nursing Diagnosis Nursing Association (NANDA), Nursing Outcomes Classification (NOC) and Nursing Classification (NIC) link (NNN link) clinical clues reasoning to determine the contents of the questionnaire were used. Then, the Delphi method to care was used. 2) The survey questionnaire was designed. The content included the clinical holistic nursing care model in breastfeeding and the model's use situation. 3) Questionnaire survey: in the national midwifery care industries, volunteers were collected who were willing to use the breastfeeding clinical holistic nursing care model in the nursing work. We issued 98 questionnaires and took back 76 valid questionnaires. Results: The construction of the breastfeeding clinical holistic nursing model includes 9 nursing diagnoses which contain the breastfeeding effective, the lack of knowledge, the decision conflicts, the risk of breast tenderness, the risk of cracked nipple, the invalid hazard occurring in breastfeeding, the risk of caregiver role strain, the risk of breast feeding jaundice and the ineffective community coping, 18 nursing outcomes and 64 nursing measures. In the composition of breastfeeding after clinical holistic nursing model in clinical practice, $\mathbf{1 0 0 \%}$ of obstetric nurses think that the breastfeeding nursing model can promote the nursing staff to care patients according to the nursing process, can help nurses to analyze, evaluate, make decision, and care about breastfeeding related problems, as well as can promote the mother's milk feeding rate. Conclusion: The establishment of the breastfeeding nursing model provides a learning material for obstetric breast-feeding. Moreover, nursing diagnosis, nursing outcomes and nursing measures correspond according to the form of chart, which are easy to use and find. The breastfeeding clinical holistic nursing model is practicing in clinical nursing, which can help nursing staff to improve the ability of nursing personnel according to evidence-based nursing patients, to improve the ability of nursing personnel analysis, evaluation, decision-making and nursing in breastfeeding problems and to promote the rate of breastfeeding. 


\section{Keywords}

\section{Breastfeeding, Clinical Global Care Model, Nursing Diagnosis, Care Outcomes, Nursing}

\section{Introduction}

In recent years, the study found that to implement the breastfeeding, to children, from the physiological level, could reduce the occurrence of gastroenteritis, otitis media, severe lower respiratory tract infections, atopic eczema and sudden infant death syndrome, etc., in infancy. In childhood, implementation of the breastfeeding that could reduce the incidence of the type 1 diabete and type 2 diabete, overweight and obesity. When they are adults, they are less plagued by chronic diseases. From the psychological level, the breastfed babies are quieter, less crying at night, and smarter. At the same time, to their mothers, implementation of the breastfeeding not only can promote the recovery of uterus and decrease the incidence of postpartum hemorrhage, but also can prevent breast cancer, ovarian cancer and osteoporosis, which make the postpartum body to recover faster [1]. Therefore, breastfeeding is good for both maternal and child's health. However, in recent years, our country breastfeeding rates show a falling trend on the whole. In 2014, Chinese exclusive breastfeeding rate of the World Bank's research results was $27.8 \%$. The city was $15.8 \%, 30.3 \%$ in the countryside. In recent years, there are a lot of reports to analyze the causes of breastfeeding decline in literature. In addition to the women, family and social factors, the survey results also showed that nursing staff lacked the ability on the impact of breast feeding factors analysis and coping. Nursing personnel is insufficient, can't make pertinent individual care plan. Breast-feeding advice and care takes a long work time; it lacks job evaluation index system; nursing workload calculation pay is out of step with income, etc. The presence of these elements will affect the implementation of the quality of obstetric care and breastfeeding. And in gynecology and obstetrics nursing teaching material list, only one "breastfeeding is invalid" breastfeeding related nursing diagnosis [1] cannot satisfy the nurses and nursing students at the present stage in China according to the nursing process to breastfeed needs of clinical evidence-based nursing.

\section{Research Methods}

\subsection{Use of NNN Links to Breastfeed Clinical Development of Holistic Nursing Model}

1) To assess breast-feeding for background information: according to the literature survey, clinical observation and textbook evaluation content determine maternal background information. Background information including lifestyle, parity, parity, physical health, breastfeeding confidence, consciously lactation, educational level, economic status, occupation, mother role adaptation, health education, care model, family and social support as well as nurses, mothers and caregivers of knowledge, attitudes, behavior and will affect the health of babies breastfed implementation. 2) To determine the nursing diagnosis: to determine the conflict of breastfeeding, lack of knowledge, effective decision-making, the risk of breast pain and the risk of cracked nipple, the risk of breastfeeding is invalid, caregiver role strain of risk, the risk of breast fed raises a gender jaundice and community in the face of the invalid, etc. 9. 3) The determined nursing outcome has 18. 4) The determined nursing measures are 64 items. 5) Set by the nursing diagnosis and nursing outcomes and nursing measures of the combination of expert consultation questionnaire breastfeeding clinical holistic nursing model.

\subsection{For Expert Advice}

In this study, we choose the obstetric nursing experts who have worked more than 10 years. The first round, 25 questionnaires are returned. There are 23 valid questionnaires. In the second round of expert consultation, we choose 23 experts to consult who participate in the first round on a high enthusiasm. The 23 experts include 4 head nurses, 5 midwives and nursing teaching, 5 midwives and 9 obstetric nurses. Their titles include 2 high, 9 deputy and 12 intermediate. Their degrees contain 3 masters and 20 undergraduates. According to the expert advice to revise breastfeeding clinical path in the fourth round of survey, all experts no longer change their opinion. As a result, experts to collect process stop after the fourth round. 


\subsection{In Clinical Nursing Practice}

Many volunteers in obstetric nursing staff in the country in the clinical nursing used in breastfeeding the whole nursing model require to fill in the questionnaire after feedback clinical effect. Volunteers aged between 25 and 50 years of fixed working were 3 - 25 years; Title: orthometric height, 5; the subtropical, high 19 people; intermediate, 32 people; primary, 20 people; education: 4 masters, 45 undergraduates, 27 specialty people. Out 98 questionnaires, 76 effective questionnaires were taken back.

\subsection{Data Collection and Analysis}

Spss13.0 statistical software for data analysis was used.

\section{The Results}

\subsection{Experts Situation Analysis}

This research adopts the expert consultation method for consulting clinical path of breastfeeding. Through statistical analysis: experts coefficient is 0.812 ; the variation coefficient is 0.748 ; four rounds of expert consultation questionnaire recovery efficiency were $92 \%, 100 \%, 100 \%, 100 \%$, accord with the requirement of Delphi expert consultation has the reliability of the research.

\subsection{Breastfeeding Clinical Index System of the Whole Nursing Model}

NNN links were used to make breastfeeding clinical nursing model as a whole. Determine the nursing diagnosis of nine, ending 18, 64 nursing measures, will form the nursing diagnosis, nursing outcome, nursing measures according to the chart in the form of one to one correspondence, in order to using simply and conveniently.

\subsection{Clinical Practice Analysis of the Clinical Nursing Model of Breastfeeding}

Table 1 shows: $100 \%$ of obstetric nursing staff think that the establishment of breastfeeding nursing model can improve the ability of nursing personnel according to the holistic nursing process nursing patients, to improve the ability of nursing personnel analysis, evaluation, decision-making and nursing in breastfeeding problems and to promote the rate of breastfeeding.

Table 2 shows: $86.8 \%$ of the obstetric nursing staff apply breast feeding clinical holistic nursing model to the nursing interns in the specialty of knowledge learning. $76.3 \%$ of the obstetric nursing staff apply it to the new nursing staff in professional training. $68.4 \%$ of the obstetric nursing staff recommend it as learning materials to nursing staff. $6.5 \%$ of the obstetric nursing staff use it to develop the maternal breastfeeding care plan.

Table 1. Breastfeeding clinical holistic nursing model's clinical practice effect.

\begin{tabular}{|c|c|c|c|}
\hline Content & $\mathrm{N}$ & $\%$ & $\%$ \\
\hline $\begin{array}{l}\text { Problem analysis, evaluation, decision-making, and } \\
\text { care ability in breastfeeding }\end{array}$ & 76 & $100 \%$ & 0 \\
\hline $\begin{array}{l}\text { The ability that can care patients according to the } \\
\text { nursing procedure }\end{array}$ & 76 & $100 \%$ & 0 \\
\hline To improve the breastfeeding rates & 76 & $100 \%$ & 0 \\
\hline
\end{tabular}

Table 2. Breastfeeding clinical holistic nursing model’s clinical practice approach.

\begin{tabular}{lll}
\hline Approach & $\mathrm{N}$ & $\%$ \\
\hline The specialized knowledge training nurses & 66 & $86.8 \%$ \\
The specialized knowledge training new nurses & 58 & $66.3 \%$ \\
Nursing staff professional work learning materials & 52 & $6.4 \%$ \\
Developing a plan of the individual patient care & 5 & $6.5 \%$ \\
\hline
\end{tabular}




\section{Discussion}

\subsection{The Significance of Developing Clinical Holistic Nursing Model}

About the significance of this topic, research mainly reflects in the following three points: 1) In gynecology and obstetrics nursing textbooks at present, only listed one "breastfeeding is invalid" breastfeeding related nursing diagnosis cannot meet the needs of the nursing problems of evidence-based nursing process breastfeeding according to the present stage in our country. Clinical study of this subject is to establish breastfeeding the whole nursing model and supplement the shortage of the textbook, as resources for nursing students in learning, the teacher in the teaching, the nurse in clinical nursing work, to make plans for the individual. 2) The 201 items nursing diagnoses listed in the basic nursing teaching material only have names and have no description to the definition of nursing diagnosis, so that nursing students, teachers, clinical nursing staff in use process can't grasp the practical significance of the nursing diagnosis well and feel very abstract and difficult to apply. At the same time, the corresponding nursing diagnosis suggested that nursing outcome did not introduce [2]. Therefore, it cannot be used as reference materials in working out nursing plan, which makes our country facing the bottleneck problem in nursing diagnosis, outcomes and measures. With the continuous deepening of the study of the clinical pathway of multi-diseases, to form a perfect clinical nursing model database can be regarded as a breakthrough in the bottleneck problem in using of nursing diagnosis in our country at present, and can form a unique knowledge system in the field of nursing specialty suitable for China's national conditions [3]. 3) The nursing staff can make use of a computer system, establish breastfeeding clinical holistic nursing model database in evaluating patients on the basis of background story, determine the individual care plan and improve the efficiency of nursing work [4].

\subsection{Use NNN Links to Breastfeed Clinical Feasibility of the Whole Nursing Model Developed}

NNN link refers to North America, NANDA nursing association, nursing diagnosis, nursing outcomes classification (NOC) and nursing intervention classification (NIC) links. Developed for clinical use of NNN link, the whole nursing model advantages are: 1) Helping nursing staff to build the optimal nursing diagnosis, the optimal nursing intervention, and the optimal nursing outcome of combining the knowledge of the system. 2) Promoting nursing students using the north American association of nursing diagnosis nursing diagnosis classification, the classification of the nursing measures and nursing outcomes classification of nursing language in the teaching practice [1]. In Dr. Wu's book, the book also mentions that using NNN links can help nursing students to master the skills necessary for clinical decision making. Nursing students can link the database when they develop nursing plans for patients. With the development of nursing knowledge, we need to evaluate the effectiveness of different nursing measures and to choose the appropriate nursing measures to solve the diagnosis or to achieve the specific outcome of the decision-making process. Through the empirical study, it is helpful to establish the theory of nursing in the middle level, and establish the special knowledge system in the field of nursing specialty [5]. In this study, we use NNN links, experts consultation and clinical practice to make an empirical study. This research is to study the relationship between breastfeeding related nursing diagnosis, outcome and measures as well as the environment, health care organization structure, nursing process and patient outcomes, so as to establish the clinical nursing model database of breast feeding. Research is feasible.

\subsection{This Study Make Breastfeeding Clinical Holistic Nursing Model and the Differences of Clinical Nursing Path Analysis of Clinical}

Clinical nursing pathway is a time schedule for specific patient groups, with time as the horizontal axis, with admission guidance, inspection, medication, treatment, nursing, diet guidance, events, health education, discharge planning, ideal nursing measures for the vertical axis, which describes the patients care goals in detailed including when the patients need to do what kinds of care measures, when leave hospital and so on. In the course of clinical nursing, the plan is hung on the side of the patient's bed and the nursing staff execute measures in schedule corresponding to column tick. Both nurses and patients promote the completion of the nursing plan [6]. Clinical holistic nursing model developed in this study is in accordance with the nursing procedure model, and a clinical holistic nursing model for specific patient groups. The model, which is on the basis of the background of patients and on the core of nursing diagnosis, is to make sure the best nursing care and the best 
outcome. The results of this research can be used as a nursing model in clinical nursing work and teaching and it is helpful for nursing staff and nursing students when combining with the clinical cases to carry out evidences and make a individual nursing plan.

\subsection{The Index Analysis}

The clinical pathway in breastfeeding includes three indicators: nursing diagnosis, nursing outcomes and nursing measures, with reference to the editor in chief being Dr.Wu Yuan Jianyun. 1) There are 9 establishment of the nursing diagnosis. Breastfeeding, lack of knowledge, effective decision-making conflicts, in danger of caregiver role strain, community in the face of the invalid and five nursing diagnosis with the corresponding evaluation content are directly used in the editor Dr Wu's book. To "invalid hazard occurring in breastfeeding" of nursing diagnosis, which are used "ineffective breast feeding” in Dr. Wu's book and obstetrics and gynecology nursing textbooks. But in this study, through clinical evidence-based, we thought that "ineffective breast feeding" as a potential nursing problem did not occur in all women in the process of implementing the breastfeeding. The purpose of nursing is to realize the breastfeeding. According to the theory of behavioral psychologist Skinner, "Behavior is shaped by the positive or negative reinforcement." What is more, the positive reinforcement is more effective in shaping behavior [7]. Therefore, in this study, we change the name of invalid nursing diagnosis name of breast feeding to "invalid hazard occurring in breastfeeding" that is more to strengthen the awareness of nursing staff and nursing students on the implementation of breastfeeding. It is easy to occur breast tenderness, cracked nipples and breast milk jaundice feed raises a gender, which affects maternal breastfeeding confidence and affects the secretion of milk, decreases the rate of breastfeeding, therefore, increases "the risk of breast tenderness", "the risk of cracked nipple" and "the risk of breast fed raises a gender jaundice", which improve the awareness of clinical nursing staff and nursing students to prevent risks and to improve the rate of pure breast feeding. 2) The establishment of the nursing outcome has 18 items, among them, some nursing outcomes in reference to the definition on the basis of Dr Wu's book have made some changes, such as the definition of breastfeeding to establish, defined as in the book "three weeks before the breastfeeding, baby get nutrition". Baby gets nutrition through biting and sucking the breast. Experts agree that the concept is not easy to understand. Therefore, it will be defined to "postpartum breastfeeding is 3 weeks window period, the baby can get nutrition". The baby can get nutrition through biting and sucking the breast, which has been approbated by experts. 3) The establishment of the nursing measures has 64 items, in the first round of the experts consultation table, for nursing measures only lists the directory, expert advice should specifically list the implementation methods of nursing measures, for reference, therefore, in the second round consultation table lists the corresponding nursing measures of the implementation of the method get the approbation of the experts, and further perfect.

\subsection{The Situation Analysis of Clinical Practice}

Through the analysis of the research result that obstetric nurses use the breastfeeding clinical holistic nursing model in nursing clinical, $100 \%$ obstetric clinical nursing staff think that the establishment of breastfeeding clinical holistic nursing model provides a database for obstetric breast-feeding care. What is more, nursing diagnosis, nursing outcome and nursing measures can correspond according to the form chart, which facilitates to use and search. $100 \%$ of obstetric nurses think that the breastfeeding nursing model can promote the nursing staff to care patients according to the nursing process, can help nurses to analyze, evaluate, make decision, and care about breastfeeding related problems, as well as can promote the mother's milk feeding rate. The model's application of the way in the clinical nursing work and study is that $86.8 \%$ of the obstetric nurses put the breastfeeding clinical holistic nursing model applied to specialized knowledge learning in nursing interns, that $76.3 \%$ of the obstetric nurses put it applied to specialized knowledge training in the new nursing staff, that $68.4 \%$ of the obstetric nursing staff suggest that it can become learning materials for nursing staff, and that $6.5 \%$ of nursing staff use it to make maternal breastfeeding care plans. The model applied to the clinical nursing work and study obtains satisfactory nursing effect and achieves the exclusive breastfeeding.

\section{Conclusion}

Establishment of the breastfeeding nursing model provides a learning material for obstetric breast-feeding. Moreover, nursing diagnosis, nursing outcomes and nursing measures correspond according to the form of chart, 
which are easy to use and find. The breastfeeding clinical holistic nursing model is practicing in clinical nursing, which can help nursing staff to establish overall concept of holistic nursing, to improve the ability of nursing personnel according to evidence-based nursing patients, to improve the ability of nursing personnel analysis, evaluation, decision-making and nursing in breastfeeding problems, to promote the rate of breastfeeding and to promote the quality of obstetric care.

\section{Fund Project}

The educational reform projects were funded in Shanxi Province, 2012(6)-J036.

\section{References}

[1] Zheng, X.X. (2012) Gynecology and Obstetrics Nursing. People’s Medical Publishing House, Beijing.

[2] Jiang, A.L. (2012) New Basic Nursing. People’s Medical Publishing House, Beijing, 180-201.

[3] Wu, D.M., Wang, W.L., et al. (2009) The Nursing Diagnosis of Literature Review and Analysis of 10 Years. Journal of Nursing Research, 23, 1526-1527. http://www.cnki.com.cn/Article/CJFDTotal-SXHZ200917016.htm

[4] Wei, F.M., Wei, F.Q., et al. (2011) The Research Progress of the Nursing Process. Journal of Nursing, 18, 36-37. http://d.wanfangdata.com.cn/Periodical/nfhlxb201121010

[5] Wu, J.Y. (2010) Nursing Diagnosis, Outcome and Measures. Beijing Medical University Press, Beijing, 19-389.

[6] Xue, L.S., Yang, X.Y. et al. (2001) The Research on the Management of Clinical Nursing Path in Japan. Foreign Medical: Nursing Pathology, 20, 547-549. http://www.cnki.com.cn/Article/CJFDTotal-GWHL200112001.htm

[7] The DK Press (2014) Encyclopedia of Psychology. Xu, Y., Ed., Electronic Industry Press, Beijing, 82-85. 